\title{
A NOTE ON SPECIAL SMARANDACHE CURVES IN THE NULL CONE $Q^{3}$
}

\author{
F. Almaz, M.A. KülahcI
}

ABstract. As it is well-known, the geometry of curve in three-dimensions is actually characterized by Frenet vectors. In this paper, we obtain Smarandache curves by using cone frame formulas in null cone $\mathbf{Q}^{3}$. Also, we give an example related to these curves.

2010 Mathematics Subject Classification: 53A40, 53A35.

Keywords: smarandache curve, asymptotic orthonormal frame, null cone.

\section{INTRODUCTION}

Human being were bewitched by curves and curved shapes long before they took into account them as mathematical objects. But the greatest effect in the research of curves was, of course, the discovery of the calculus. Geometry before calculus includes only the simplest curves.

Smarandache geometry is a geometry which has at least one Smarandachely denied axiom [4]. An axiom is said to be Smarandachely denied, if it behaves in at least two different ways within the same space. Smarandache curve is defined as a regular curve whose position vector is composed by Frenet frame vectors of another regular curve.

The popularity of Smarandache curves in various ambient spaces have been classfied in [1]-[7], [13]-[16]. In this study, we define special Smarandache curves such as $x \alpha \beta, x \beta y, x \alpha y, \alpha \beta y$-Smarandache curves according to asymptotic orthonormal frame in the null cone $\mathbf{Q}^{3}$ and we examine the curvatures and the asymptotic orthonormal frame's vectors of Smarandache curves. We also give an example related to these curves. 


\section{Preliminaries}

Some basics of the curves in the null cone are provided from, [8]-[9].

Let $E_{1}^{4}$ be the 4 -dimensional pseudo-Euclidean space with the

$$
\widetilde{g}(X, Y)=\langle X, Y\rangle=x_{1} y_{1}+x_{2} y_{2}+x_{3} y_{3}-x_{4} y_{4}
$$

for all $X=\left(x_{1}, x_{2}, x_{3}, x_{4}\right) Y=\left(y_{1}, y_{2}, y_{3}, y_{4}\right) \in E_{1}^{4} . E_{1}^{4}$ is a flat pseudo-Riemannian manifold of signature $(3,1)$.

Let $M$ be a submanifold of $E_{1}^{4}$. If the pseudo-Riemannian metric $\widetilde{g}$ of $E_{1}^{4}$ induces a pseudo-Riemannian metric $g$ (respectively, a Riemannian metric, a degenerate quadratic form) on $M$, then $M$ is called a timelike( respectively, spacelike, degenerate) submanifold of $E_{1}^{3}$. Let $c$ be a fixed point in $E_{1}^{4}$. The pseudo-Riemannian lightlike cone(quadric cone ) is defined by

$$
\mathbf{Q}_{1}^{3}(c)=\left\{x \in E_{1}^{4}: g(x-c, x-c)=0\right\},
$$

where the point $c$ is called the center of $\mathbf{Q}_{1}^{3}(c)$. When $c=0$, we simply denote $\mathbf{Q}_{1}^{3}(0)$ by $\mathbf{Q}^{3}$ be and call it the null cone.

Let $E_{1}^{4}$ be 4 -dimensional Minkowski space and $\mathrm{Q}^{3}$ the lightlike cone in $E_{1}^{4}$. A vector $V \neq 0$ in $E_{1}^{4}$ is called spacelike, timelike or lightlike, if $\langle V, V\rangle>0,\langle V, V\rangle<0$ or $\langle V, V\rangle=0$, respectively. The norm of a vector $x \in E_{1}^{4}$ is given by $\|x\|=\sqrt{\langle x, x\rangle}$, $[12]$.

We assume that curve $x: I \rightarrow \mathbf{Q}^{3} \subset E_{1}^{4}$ is a regular curve in $\mathbf{Q}^{3}$ for $t \in I$. In the following, we always assume that the curve is regular. if

A frame field $\{x, \alpha, \beta, y\}$ on $E_{1}^{4}$ is called an asymptotic orthonormal frame field,

$$
\begin{aligned}
& \langle x, x\rangle=\langle y, y\rangle=\langle x, \alpha\rangle=\langle y, \alpha\rangle=\langle\beta, \alpha\rangle=\langle y, \beta\rangle=\langle x, \beta\rangle=0 \\
& \langle x, y\rangle=\langle\alpha, \alpha\rangle=\langle\beta, \beta\rangle=1
\end{aligned}
$$

Using $x^{\prime}(s)=\alpha(s)$ we know that $\{x(s), \alpha(s), \beta(s), y(s)\}$ from an asymptotic orthonormal frame along the curve $x(s)$ and the cone frenet formulas of $x(s)$ are given by

$$
\begin{aligned}
x^{\prime}(s) & =\alpha(s) \\
\alpha^{\prime}(s) & =\kappa(s) x(s)-y(s) \\
\beta^{\prime}(s) & =\tau(s) x(s) \\
y^{\prime}(s) & =-\kappa(s) \alpha(s)-\tau(s) \beta(s)
\end{aligned}
$$

where the functions $\kappa(s)$ and $\tau(s)$ are called cone curvature functions of the curve $x(s),[10]$. 
Let $x: I \rightarrow \mathbf{Q}^{3} \subset E_{1}^{4}$ be a spacelike curve in $\mathbf{Q}^{3}$ with an arc length parameter $s$. Then $x=x(s)=\left(x_{1}, x_{2}, x_{3}, x_{4}\right)$ can be written as

$$
x(s)=\frac{1}{2 \sqrt{f_{s}^{2}+g_{s}^{2}}}\left(2 f, 2 g, 1-f^{2}-g^{2}, 1+f^{2}+g^{2}\right)
$$

for some non constant function $f(s)$ and $g(s),[11]$.

\section{Smarandache Curves in The Null Cone $\mathbf{Q}^{3}$}

In this section, we define binary Smarandache curves according to the asymptotic orthonormal frame in $\mathbf{Q}^{3}$. Also, we obtain the asymptotic orthonormal frame and cone curvature functions of the Smarandache partners lying on $\mathbf{Q}^{3}$ using cone frenet formulas.

Smarandache curve $\gamma=\gamma\left(s^{*}(s)\right)$ of the curve $x$ is a regular unit speed curve lying fully on $\mathbf{Q}^{3}$. Let $\{x, \alpha, \beta, y\}$ and $\left\{\gamma, \alpha_{\gamma}, \beta_{\gamma}, y_{\gamma}\right\}$ be the moving asymptotic orthonormal frames of $x$ and $\gamma$, respectively.

Definition 1. Let $x$ be unit speed spacelike curve lying on $\mathbf{Q}^{3}$ with the moving asymptotic orthonormal frame $\{x, \alpha, \beta, y\}$. Then, $x \alpha \beta-$ Smarandache curve of $x$ is defined by

$$
\gamma_{x \alpha \beta}\left(s^{*}\right)=\frac{1}{\sqrt{b^{2}+c^{2}}}(a x(s)+b \alpha(s)+c \beta(s)),
$$

where $a, b, c \in \mathbb{R}_{0}^{+}$.

Theorem 1. Let $x$ be unit speed spacelike curve in $\mathbf{Q}^{3}$ with the moving asymptotic orthonormal frame $\{x, \alpha, \beta, y\}$ and cone curvatures $\kappa(s), \tau(s)$ and let $\gamma_{x \alpha \beta}$ be $x \alpha \beta-$ Smarandache curve with asymptotic orthonormal frame $\left\{\gamma_{x \alpha \beta}, \alpha_{x \alpha \beta}, \beta_{x \alpha \beta}, y_{x \alpha \beta}\right\}$. Then the following relations hold:

i) The asymptotic orthonormal frame $\left\{\gamma_{x \alpha \beta}, \alpha_{x \alpha \beta}, \beta_{x \alpha \beta}, y_{x \alpha \beta}\right\}$ of the $x \alpha \beta$-Smarandache curve $\gamma_{x \alpha \beta}$ is given as

$$
\left[\begin{array}{c}
\gamma_{x \alpha \beta} \\
\alpha_{x \alpha \beta} \\
\beta_{x \alpha \beta} \\
y_{x \alpha \beta}
\end{array}\right]=\left[\begin{array}{cccc}
\frac{a}{\sqrt{b^{2}+c^{2}}} & \frac{b}{\sqrt{b^{2}+c^{2}}} & \frac{c}{\sqrt{b^{2}+c^{2}}} & 0 \\
\frac{b \kappa+c \tau}{\Psi} & \frac{a}{\Psi} & 0 & \frac{-b}{\Psi} \\
B_{1} & B_{2} & B_{3} & B_{4} \\
\Upsilon_{1} & \Upsilon_{2} & \Upsilon_{3} & \Upsilon_{4}
\end{array}\right]\left[\begin{array}{c}
x \\
\alpha \\
\beta \\
y
\end{array}\right],
$$


where

$$
\begin{aligned}
\Psi & =\sqrt{a^{2}-2 b(b \kappa+c \tau)}, w=\frac{\Psi}{\sqrt{b^{2}+c^{2}}} \\
A_{1} & =\frac{b \kappa+c \tau}{\Psi}, A_{2}=\frac{a}{\Psi}, A_{3}=\frac{1}{w}
\end{aligned}
$$

and

$$
\begin{aligned}
& B_{1}=\frac{\sqrt{b^{2}+c^{2}}}{\Psi^{3}}\left(\Psi^{\prime}(b \kappa+c \tau)+\Psi\left(b \kappa^{\prime}+c \tau^{\prime}\right)+a \Psi \kappa\right) \\
& B_{2}=\frac{\sqrt{b^{2}+c^{2}}}{\Psi^{3}}\left(\Psi(b \kappa+c \tau)-a-\Psi \kappa \sqrt{b^{2}+c^{2}}\right) \\
& B_{3}=\frac{-\tau\left(b^{2}+c^{2}\right)}{\Psi^{2}}, B_{4}=\frac{\sqrt{b^{2}+c^{2}}}{\Psi^{3}}\left(-a \Psi+\sqrt{b^{2}+c^{2}}\right) ; \\
& \Upsilon_{1}=-B_{1}-\frac{a D}{2 \sqrt{b^{2}+c^{2}}}, \Upsilon_{2}=-B_{2}-\frac{b D}{2 \sqrt{b^{2}+c^{2}}}, \Upsilon_{3}=-B_{3}-\frac{c D}{2 \sqrt{b^{2}+c^{2}}}, \\
& \Upsilon_{4}=-B_{4}
\end{aligned}
$$

and

$$
D=2 \frac{\left(A_{1}^{\prime}+\kappa A_{2}\right)\left(-A_{2}+A_{3}^{\prime}\right)}{w^{2}}+\frac{1}{w^{2}}\left(A_{1}+A_{2}^{\prime}-\kappa A_{3}\right)^{2}+\left(\tau A_{3}\right)^{2}
$$

or

$$
\begin{aligned}
D= & \frac{\left(b^{2}+c^{2}\right)}{\Psi^{6}}\left(-2\left(\Psi^{\prime}(b \kappa+c \tau)+\Psi\left(b \kappa^{\prime}+c \tau^{\prime}\right)+a \Psi \kappa\right)\left(a \Psi+\sqrt{b^{2}+c^{2}}\right)\right. \\
& \left.+\left(\Psi(b \kappa+c \tau)-a-\Psi \kappa \sqrt{b^{2}+c^{2}}\right)^{2}\right)+\frac{\tau^{2}\left(b^{2}+c^{2}\right)}{\Psi^{2}}
\end{aligned}
$$

ii) The cone curvatures $\kappa_{\gamma_{x \alpha \beta}}\left(s^{*}\right)$ and $\tau_{\gamma_{x \alpha \beta}}\left(s^{*}\right)$ of the curve $\gamma_{x \alpha \beta}$ is given by

$$
\begin{gathered}
\kappa_{\gamma_{x \alpha \beta}}\left(s^{*}\right)=-\frac{D}{2} \\
\tau_{\gamma_{x \alpha \beta}}\left(s^{*}\right)=\sqrt{2\left(\Upsilon_{1}-\kappa^{\prime}\right) \Upsilon_{4}+\left(\Upsilon_{2}-\kappa\right)^{2}+\Upsilon_{3}^{2}-\kappa_{\gamma_{x \alpha \beta}}^{2}},
\end{gathered}
$$

where

$$
s^{*}=\frac{1}{\sqrt{b^{2}+c^{2}}} \int \sqrt{a^{2}-2 b(b \kappa+c \tau)} d s .
$$


Proof. i) We assume that the curve $x$ is a unit speed spacelike curve with the asymptotic orthonormal frame $\{x, \alpha, \beta, y\}$ and cone curvatures $\kappa, \tau$. Differentiating the equation (3.1) with respect to $s$ and considering (2.1), we have

$$
\gamma_{x \alpha \beta}^{\prime}\left(s^{*}\right)=\left(\frac{a}{\Psi}\right) \alpha(s)+\left(\frac{b \kappa+c \tau}{\Psi}\right) x(s)+\left(\frac{1}{w}\right) y(s),
$$

where

$$
\begin{aligned}
& w=\frac{d s^{*}}{d s}=\frac{1}{\sqrt{b^{2}+c^{2}}} \sqrt{a^{2}-2 b(b \kappa+c \tau)}, \\
& \Psi=\sqrt{a^{2}-2 b(b \kappa+c \tau)} .
\end{aligned}
$$

It can be easily seen that the tangent vector $\gamma_{x \alpha \beta}^{\prime}\left(s^{*}\right)=\alpha_{x \alpha \beta}\left(s^{*}\right)$ is a unit spacelike vector.

Differentiating (3.7), we obtain equation as follows

$$
\gamma_{x \alpha \beta}^{\prime \prime}\left(s^{*}\right)=B_{1} x(s)+B_{2} \alpha(s)+B_{3} \beta(s)+B_{4} y(s),
$$

where $B_{1}=\frac{A_{1}^{\prime}+\kappa A_{2}}{w}, B_{2}=\frac{A_{1}+A_{2}^{\prime}-\kappa A_{3}}{w}, B_{3}=\frac{-\tau A_{3}}{w}, B_{4}=\frac{-A_{2}+A_{3}^{\prime}}{w}$.

$$
y_{x \alpha \beta}\left(s^{*}\right)=-\gamma_{x \alpha \beta}^{\prime \prime}-\frac{1}{2}\left\langle\gamma_{x \alpha \beta}^{\prime \prime}, \gamma_{x \alpha \beta}^{\prime \prime}\right\rangle \gamma_{x \alpha \beta}
$$

By the help of previous equation (3.11), we obtain

$$
y_{x \alpha \beta}\left(s^{*}\right)=\Upsilon_{1} x(s)+\Upsilon_{2} \alpha(s)+\Upsilon_{3} \beta(s)+\Upsilon_{4} y(s),
$$

where $\Upsilon_{1}=-B_{1}-\frac{a D}{2 \sqrt{b^{2}+c^{2}}}, \Upsilon_{2}=-B_{2}-\frac{b D}{2 \sqrt{b^{2}+c^{2}}}, \Upsilon_{3}=-B_{3}-\frac{c D}{2 \sqrt{b^{2}+c^{2}}}, \Upsilon_{4}=-B_{4}$.

ii) Using equations $\kappa_{\gamma_{x \alpha \beta}}\left(s^{*}\right)=-\frac{1}{2}\left\langle\gamma_{x \alpha \beta}^{\prime \prime}, \gamma_{x \alpha \beta}^{\prime \prime}\right\rangle$ and

$$
\tau_{\gamma_{x \alpha \beta}}^{2}\left(s^{*}\right)=\left\langle x^{\prime \prime \prime}-\kappa \alpha-\kappa^{\prime} x, x^{\prime \prime \prime}-\kappa \alpha-\kappa^{\prime} x\right\rangle-\kappa_{\gamma_{x \alpha \beta}}^{2}\left(s^{*}\right) .
$$

The curvatures $\kappa_{\gamma_{x \alpha \beta}}\left(s^{*}\right)$ and $\tau_{\gamma_{x \alpha \beta}}\left(s^{*}\right)$ of the $\gamma_{x \alpha \beta}\left(s^{*}\right)$ are explicity obtained by

$$
\begin{gathered}
\kappa_{\gamma_{x \alpha \beta}}\left(s^{*}\right)=-\frac{1}{2} D \\
\tau_{\gamma_{x \alpha \beta}}^{2}\left(s^{*}\right)=2\left(\Upsilon_{1}-\kappa^{\prime}\right) \Upsilon_{4}+\left(\Upsilon_{2}-\kappa\right)^{2}+\Upsilon_{3}^{2}-\kappa_{\gamma_{x \alpha \beta}}^{2} .
\end{gathered}
$$

Thus, the theorem is proved. 
Definition 2. Let $x$ be unit speed spacelike curve lying on $\mathbf{Q}^{3}$ with the moving asymptotic orthonormal frame $\{x, \alpha, \beta, y\}$. Then, $x \beta y$-smarandache curve of $x$ is defined by

$$
\gamma_{x \beta y}\left(s^{*}\right)=\frac{1}{\sqrt{2 a c+b^{2}}}(a x(s)+b \beta(s)+c y(s)),
$$

where $a, b . c \in \mathbb{R}_{0}^{+}$.

Theorem 2. Let $x$ be unit speed spacelike curve in $\mathbf{Q}^{3}$ with the moving asymptotic orthonormal frame $\{x, \alpha, \beta, y\}$ and cone curvatures $\kappa(s), \tau(s)$ and let $\gamma_{x \beta y}$ be $x \beta y-$ Smarandache curve with asymptotic orthonormal frame $\left\{\gamma_{x \beta y}, \alpha_{x \beta y}, \beta_{x \beta y}, y_{x \beta y}\right\}$. Then the following relations hold:

i) The asymptotic orthonormal frame $\left\{\gamma_{x \beta y}, \alpha_{x \beta y}, \beta_{x \beta y}, y_{x \beta y}\right\}$ of the $x \beta y$-Smarandache curve $\gamma_{x \beta y}$ is given as

$$
\left[\begin{array}{c}
\gamma_{x \beta y} \\
\alpha_{x \beta y} \\
\beta_{x \beta y} \\
y_{x \beta y}
\end{array}\right]=\left[\begin{array}{cccc}
\frac{a}{\sqrt{2 a c+b^{2}}} & 0 & \frac{b}{\sqrt{2 a c+b^{2}}} & \frac{c}{\sqrt{2 a c+b^{2}}} \\
\frac{b \tau}{\eta} & \frac{a-c \kappa}{\eta} & \frac{-c \tau}{\eta} & 0 \\
B_{1}^{*} & B_{2}^{*} & B_{3}^{*} & B_{4}^{*} \\
\omega_{1} & \omega_{2} & \omega_{3} & \omega_{4}
\end{array}\right]\left[\begin{array}{c}
x \\
\alpha \\
\beta \\
y
\end{array}\right],
$$

where

$$
\begin{aligned}
\eta & =\sqrt{a^{2}-2 a c+c^{2}\left(\kappa^{2}+\tau^{2}\right)}, \omega=\frac{\eta}{\sqrt{2 a c+b^{2}}}=\frac{d s^{*}}{d s} ; \\
A_{1} & =\frac{b \tau}{\eta}, A_{2}=\frac{a-c \kappa}{\eta}, A_{3}=\frac{-c \tau}{\eta} ; \\
B_{1}^{*} & =\frac{A_{1}^{\prime}+\kappa A_{2}+\tau A_{3}}{w}, B_{2}^{*}=\frac{A_{1}+A_{2}^{\prime}}{w}, B_{3}^{*}=\frac{A_{3}^{\prime}}{w}, B_{4}^{*}=\frac{-A_{2}}{w}
\end{aligned}
$$

and

$$
\begin{aligned}
\omega_{1} & =-B_{1}^{*}-\frac{a T}{2 \sqrt{2 a c+b^{2}}}, \omega_{2}=-B_{2}^{*}, \omega_{3}=-B_{3}^{*}-\frac{b T}{2 \sqrt{2 a c+b^{2}}}, \\
\omega_{4} & =-B_{4}^{*}-\frac{c T}{2 \sqrt{2 a c+b^{2}}}, \\
T & =\frac{1}{\omega^{2}}\left(-2 A_{2}\left(A_{1}^{\prime}+\kappa A_{2}+\tau A_{3}\right)+\left(A_{1}+A_{2}^{\prime}\right)^{2}+A_{3}^{\prime 2}\right) .
\end{aligned}
$$

or

$$
T=\frac{2 a c+b^{2}}{\eta}\left(\begin{array}{c}
\frac{2(c \kappa-a)}{\eta^{3}}\left(b\left(\tau^{\prime} \eta-\tau \eta^{\prime}\right)+\eta\left(\kappa a-c\left(\kappa^{2}+\tau^{2}\right)\right)\right) \\
+\frac{1}{\eta^{4}}\left(\left(\eta(b \tau-c \kappa)+\eta^{\prime}(a-c \kappa)\right)^{2}+\frac{c^{2}\left(\tau \eta^{\prime}-\tau^{\prime} \eta\right)^{2}}{\eta}\right)
\end{array}\right)
$$


F. Almaz, M.A. Külahci - A Note on Special Smarandache Curves ...

ii) The cone curvature $\kappa_{\gamma_{x \beta y}}\left(s^{*}\right)$ and $\tau_{\gamma_{x \beta y}}\left(s^{*}\right)$ of the curve $\gamma_{x \beta y}$ is given by

$$
\begin{gathered}
\kappa_{\gamma_{x \beta y}}\left(s^{*}\right)=\frac{-T}{2}, \\
\tau_{\gamma_{x \beta y}}^{2}\left(s^{*}\right)=2\left(\omega_{1}-\kappa^{\prime}\right) \omega_{4}+\left(\omega_{2}-\kappa\right)^{2}+\omega_{3}^{2}-\kappa_{\gamma_{x \beta y}}^{2}
\end{gathered}
$$

where

$$
s^{*}=\frac{1}{\sqrt{2 a c+b^{2}}} \int \sqrt{a^{2}-2 a c+c^{2}\left(\kappa^{2}+\tau^{2}\right)} d s .
$$

Proof. i) We assume that the curve $x$ is a unit speed spacelike curve with the asymptotic orthonormal frame $\{x, \alpha, \beta, y\}$ and cone curvatures $\kappa, \tau$. Differentiating the equation (3.14) with respect to $s$ and considering (2.1), we have

$$
\gamma_{x \beta y}^{\prime}\left(s^{*}\right)=\frac{b \tau}{\eta} \vec{x}(s)+\frac{a-c \kappa}{\eta} \vec{\alpha}(s)-\frac{c \tau}{\eta} \vec{\beta}(s)
$$

or

$$
\gamma_{x \beta y}^{\prime}\left(s^{*}\right)=A_{1} \vec{x}+A_{2} \vec{\alpha}+A_{3} \vec{\beta} .
$$

By considering (3.17), we get

$$
\gamma_{x \beta y}^{\prime}\left(s^{*}\right)=\alpha(s)=\alpha_{x \beta y} .
$$

Here, it can be easily seen that the tangent vector $\vec{\alpha}_{x \beta y}$ is a unit spacelike vector. Differentiating (3.19) and using (3.17), we obtain

$$
\gamma_{x \beta y}^{\prime \prime}\left(s^{*}\right)=B_{1}^{*} \vec{x}+B_{2}^{*} \vec{\alpha}+B_{3}^{*} \vec{\beta}-B_{4}^{*} \vec{y},
$$

where $B_{1}^{*}=\frac{A_{1}^{\prime}+\kappa A_{2}+\tau A_{3}}{w}, B_{2}^{*}=\frac{A_{1}+A_{2}^{\prime}}{w}, B_{3}^{*}=\frac{A_{3}^{\prime}}{w}, B_{4}^{*}=\frac{-A_{2}}{w}$.

By the help of equation $y_{x \beta y}\left(s^{*}\right)=-\gamma_{x \beta y}^{\prime \prime}-\frac{1}{2}\left\langle\gamma_{x \beta y}^{\prime \prime}, \gamma_{x \beta y}^{\prime \prime}\right\rangle \gamma_{x \beta y}$, we write

$$
y_{x \beta y}\left(s^{*}\right)=\omega_{1} x(s)+\omega_{2} \alpha(s)+\omega_{3} \beta(s)+\omega_{4} y(s),
$$

where

$$
\begin{aligned}
& \omega_{1}=-B_{1}^{*}-\frac{a T}{2 \sqrt{2 a c+b^{2}}}, \omega_{2}=-B_{2}^{*} \\
& \omega_{3}=-B_{3}^{*}-\frac{b T}{2 \sqrt{2 a c+b^{2}}}, \omega_{4}=-B_{4}^{*}-\frac{c T}{2 \sqrt{2 a c+b^{2}}} .
\end{aligned}
$$

ii)

$$
\kappa_{\gamma_{x \beta y}}\left(s^{*}\right)=-\frac{1}{2}\left\langle\gamma_{x \beta y}^{\prime \prime}, \gamma_{x \beta y}^{\prime \prime}\right\rangle,
$$




$$
\tau_{\gamma_{x \beta y}}^{2}\left(s^{*}\right)=\left\langle\beta-\kappa \alpha-\kappa^{\prime} x, \beta-\kappa \alpha-\kappa^{\prime} x\right\rangle-\kappa_{\gamma_{x \beta y}}^{2} .
$$

By using (3.22), the curvatures $\kappa_{\gamma_{x \beta y}}\left(s^{*}\right)$ and $\tau_{\gamma_{x \beta y}}\left(s^{*}\right)$ of the $\gamma_{x \beta y}\left(s^{*}\right)$ are explicity obtained

$$
\begin{aligned}
& \kappa_{\gamma_{x \beta y}}\left(s^{*}\right)=-\frac{1}{2}\left\langle\gamma_{x \beta y}^{\prime \prime}, \gamma_{x \beta y}^{\prime \prime}\right\rangle=\frac{-T}{2}, \\
& \tau_{\gamma_{x \beta \alpha}}^{2}\left(s^{*}\right)=2\left(\omega_{1}-\kappa^{\prime}\right) \omega_{4}+\left(\omega_{2}-\kappa\right)^{2}+\omega_{3}^{2}-\kappa_{\gamma_{x \alpha}}^{2}
\end{aligned}
$$

where

$$
T=\frac{2 a c+b^{2}}{\eta}\left(\begin{array}{c}
\frac{2(c \kappa-a)}{\eta^{3}}\left(b\left(\tau^{\prime} \eta-\tau \eta^{\prime}\right)+\eta\left(\kappa a-c\left(\kappa^{2}+\tau^{2}\right)\right)\right) \\
+\frac{1}{\eta^{4}}\left(\left(\eta(b \tau-c \kappa)+\eta^{\prime}(a-c \kappa)\right)^{2}+\frac{c^{2}\left(\tau \eta^{\prime}-\tau^{\prime} \eta\right)^{2}}{\eta}\right)
\end{array}\right) .
$$

Definition 3. Let $x$ be unit speed spacelike curve lying on $\mathbf{Q}^{3}$ with the moving asymptotic orthonormal frame $\{x, \alpha, \beta, y\}$. Then, $x \alpha y-$ Smarandache curve of $x$ is defined by

$$
\gamma_{x \alpha y}\left(s^{*}\right)=\frac{1}{\sqrt{2 a c+b^{2}}}(a x(s)+b \alpha(s)+c y(s)),
$$

where $a, b, c \in \mathbb{R}_{0}^{+}$.

Theorem 3. Let $x$ be unit speed spacelike curve in $\mathbf{Q}^{3}$ with the moving asymptotic orthonormal frame $\{x, \alpha, \beta, y\}$ and cone curvatures $\kappa, \tau$ and let $\gamma_{x \alpha y}$ be xaySmarandache curve with asymptotic orthonormal frame $\left\{\gamma_{x \alpha y}, \alpha_{x \alpha y}, \beta_{x \alpha y}, y_{x \alpha y}\right\}$. Then the following relations hold:

i) The asymptotic orthonormal frame $\left\{\gamma_{x \alpha y}, \alpha_{x \alpha y}, \beta_{x \alpha y}, y_{x \alpha y}\right\}$ of the xay-Smarandache curve $\gamma_{x \alpha y}$ is given as

$$
\left[\begin{array}{c}
\gamma_{x \alpha y} \\
\alpha_{x \alpha y} \\
\beta_{x \alpha y} \\
y_{x \alpha y}
\end{array}\right]=\left[\begin{array}{cccc}
\frac{a}{\sqrt{2 a c+b^{2}}} & \frac{b}{\sqrt{2 a c+b^{2}}} & 0 & \frac{c}{\sqrt{2 a c+b^{2}}} \\
\rho_{1} & \rho_{2} & \rho_{3} & \rho_{4} \\
\frac{\rho_{1}^{\prime}+\kappa \rho_{2}+\rho_{3} \tau}{M} & \frac{\rho_{2}^{\prime}+\rho_{1}-\kappa \rho_{4}}{M} & \frac{\rho_{3}^{\prime}-\tau \rho_{4}}{M} & \frac{-\rho_{2}+\rho_{4}^{\prime}}{M} \\
-\frac{\rho_{1}^{\prime}+\kappa \rho_{2}+\rho_{3} \tau}{M}-a \phi & -\frac{\rho_{2}^{\prime}+\rho_{1}-\kappa \rho_{4}}{M}-b \phi & -\frac{\rho_{3}^{\prime}+\tau \rho_{4}}{M} & -\frac{-\rho_{2}+\rho_{4}^{\prime}}{M}-c \phi
\end{array}\right]\left[\begin{array}{l}
x \\
\alpha \\
\beta \\
y
\end{array}\right],
$$

where

$$
\begin{gathered}
\rho_{1}=\frac{b \kappa}{\Omega}, \rho_{2}=\frac{a-c \kappa}{\Omega}, \rho_{3}=\frac{-c \tau}{\Omega}, \rho_{4}=\frac{-b}{\Omega} \\
\Omega=\sqrt{a^{2}-2\left(a c+b^{2}\right) \kappa+c^{2}\left(\kappa^{2}+\tau^{2}\right)} ; M=\frac{\Omega}{\sqrt{2 a c+b^{2}}},
\end{gathered}
$$




$$
\begin{gathered}
L=\frac{1}{M^{2}}\left(2\left(\rho_{1}^{\prime}+\kappa \rho_{2}+\rho_{3} \tau\right)\left(-\rho_{2}+\rho_{4}^{\prime}\right)+\left(\rho_{2}^{\prime}+\rho_{1}-\kappa \rho_{4}\right)^{2}+\left(\rho_{3}^{\prime}-\tau \rho_{4}\right)^{2}\right), \\
\phi=\frac{L}{2 \sqrt{2 a c+b^{2}}} .
\end{gathered}
$$

ii) The cone curvatures $\kappa_{\gamma_{x \alpha y}}\left(s^{*}\right)$ and $\tau_{\gamma_{x \alpha y}}\left(s^{*}\right)$ of the curve $\gamma_{x \alpha y}$ is given by

$$
\begin{gathered}
\kappa_{\gamma_{x \alpha y}}\left(s^{*}\right)=-\frac{L}{2}, \\
\tau_{\gamma_{x \alpha y}}^{2}\left(s^{*}\right)=2\left(\frac{\rho_{1}^{\prime}+\kappa \rho_{2}+\rho_{3} \tau}{M}+a \phi+\kappa^{\prime}\right)\left(\frac{-\rho_{2}+\rho_{4}^{\prime}}{M}+c \phi\right) \\
+\left(\frac{\rho_{2}^{\prime}+\rho_{1}-\kappa \rho_{4}}{M}+b \phi+\kappa\right)^{2}+\left(\frac{\rho_{3}^{\prime}+\tau \rho_{4}}{M}\right)^{2}-\frac{L^{2}}{4}
\end{gathered}
$$

where

$$
s^{*}=\frac{1}{\sqrt{2 a c+b^{2}}} \int \sqrt{a^{2}-2\left(a c+b^{2}\right) \kappa+c^{2}\left(\kappa^{2}+\tau^{2}\right)} d s .
$$

Proof. i) Let the curve $x$ be a unit speed spacelike curve with the asymptotic orthonormal frame $\{x, \alpha, \beta, y\}$ and cone curvatures $\kappa, \tau$. Differentiating the equation (3.23) with respect to $s$ and considering (2.1), we find

$$
\gamma_{x \alpha y}^{\prime}\left(s^{*}\right) \frac{d s^{*}}{d s}=\frac{1}{\sqrt{2 a c+b^{2}}}(b \kappa \overrightarrow{x(s)}+(a-c \kappa) \overrightarrow{\alpha(s)}-c \tau \overrightarrow{\beta(s)}-b \overrightarrow{y(s)}) .
$$

This can be written as following

$$
\alpha_{x \alpha y}\left(s^{*}\right)=\frac{b \kappa}{\Omega} \overrightarrow{x(s)}+\frac{a-c \kappa}{\Omega} \overrightarrow{\alpha(s)}-\frac{c \tau}{\Omega} \overrightarrow{\beta(s)}-\frac{b}{\Omega} \overrightarrow{y(s)},
$$

where

$$
\Omega=\sqrt{a^{2}-2\left(a c+b^{2}\right) \kappa+c^{2}\left(\kappa^{2}+\tau^{2}\right)}
$$

Differentiating (3.29) and using (3.30), we get

$$
\begin{aligned}
\gamma_{x \alpha y}^{\prime \prime}= & \left(\frac{\rho_{1}^{\prime}+\kappa \rho_{2}+\rho_{3} \tau}{M}\right) x+\left(\frac{\rho_{2}^{\prime}+\rho_{1}-\kappa \rho_{4}}{M}\right) \alpha \\
& +\left(\frac{\rho_{3}^{\prime}-\tau \rho_{4}}{M}\right) \beta+\left(\frac{-\rho_{2}+\rho_{4}^{\prime}}{M}\right) y,
\end{aligned}
$$

where $\rho_{1}=\frac{b \kappa}{\Omega}, \rho_{2}=\frac{a-c \kappa}{\Omega}, \rho_{3}=\frac{-c \tau}{\Omega}, \rho_{4}=\frac{-b}{\Omega}$.

$$
y_{x \alpha y}\left(s^{*}\right)=-\gamma_{x \alpha y}^{\prime \prime}-\frac{1}{2}\left\langle\gamma_{x \alpha y}^{\prime \prime}, \gamma_{x \alpha y}^{\prime \prime}\right\rangle \gamma_{x \alpha y}, \text { and }\left\langle\gamma_{x \alpha y}^{\prime \prime}, \gamma_{x \alpha y}^{\prime \prime}\right\rangle=L \text {. }
$$


By the help of equation (3.32), we obtain

$$
\begin{aligned}
y_{x \alpha y}\left(s^{*}\right)=( & \left.-\frac{\rho_{1}^{\prime}+\kappa \rho_{2}+\rho_{3} \tau}{M}-a \phi\right) x(s)+\left(-\frac{\rho_{2}^{\prime}+\rho_{1}-\kappa \rho_{4}}{M}-b \phi\right) \alpha(s) \\
& +\left(-\frac{\rho_{3}^{\prime}+\tau \rho_{4}}{M}\right) \beta(s)+\left(-\frac{-\rho_{2}+\rho_{4}^{\prime}}{M}-c \phi\right) y(s),
\end{aligned}
$$

where

$$
\begin{aligned}
\phi= & \frac{L}{2 \sqrt{2 a c+b^{2}}}, L=\frac{1}{M^{2}}\left(2\left(\rho_{1}^{\prime}+\kappa \rho_{2}+\rho_{3} \tau\right)\left(-\rho_{2}+\rho_{4}^{\prime}\right)\right. \\
& \left.+\left(\rho_{2}^{\prime}+\rho_{1}-\kappa \rho_{4}\right)^{2}+\left(\rho_{3}^{\prime}-\tau \rho_{4}\right)^{2}\right)
\end{aligned}
$$

ii) Using (3.22), we have (3.26) and (3.27).

Definition 4. Let $x$ be unit speed spacelike curve lying on $\mathbf{Q}^{3}$ with the moving asymptotic orthonormal frame $\{x, \alpha, \beta, y\}$. Then, $\alpha \beta y$-Smarandache curve of $x$ is defined by

$$
\gamma_{\alpha \beta y}\left(s^{*}\right)=\frac{1}{\sqrt{a^{2}+b^{2}}}(a \alpha(s)+b \beta(s)+c y(s)),
$$

where $a, b, c \in \mathbb{R}_{0}^{+}$.

Theorem 4. Let $x$ be unit speed spacelike curve in $\mathbf{Q}^{3}$ with the moving asymptotic orthonormal frame $\{x, \alpha, \beta, y\}$ and cone curvatures $\kappa, \tau$ and let $\gamma_{\alpha \beta y}$ be $\alpha \beta y-$ Smarandache curve with asymptotic orthonormal frame $\left\{\gamma_{\alpha \beta y}, \alpha_{\alpha \beta y}, \beta_{\alpha \beta y}, y_{\alpha \beta y}\right\}$. Then the following relations hold:

i) The asymptotic orthonormal frame $\left\{\gamma_{\alpha \beta y}, \alpha_{\alpha \beta y}, \beta_{\alpha \beta y}, y_{\alpha \beta y}\right\}$ of the $\alpha \beta y$-Smarandache curve $\gamma_{\alpha \beta y}$ is given as

$$
\left[\begin{array}{c}
\gamma_{\alpha \beta y} \\
\alpha_{\alpha \beta y} \\
\beta_{\alpha \beta y} \\
y_{\alpha \beta y}
\end{array}\right]=\left[\begin{array}{cccc}
0 & \frac{a}{\sqrt{a^{2}+b^{2}}} & \frac{b}{\sqrt{a^{2}+b^{2}}} & \frac{c}{\sqrt{a^{2}+b^{2}}} \\
A_{1} & A_{2} & A_{3} & A_{4} \\
C_{1} & C_{2} & C_{3} & C_{4} \\
-C_{1} & -C_{2}-\frac{a M}{2 \sqrt{a^{2}+b^{2}}} & -C_{3}-\frac{b M}{2 \sqrt{a^{2}+b^{2}}} & -C_{4}-\frac{c M}{2 \sqrt{a^{2}+b^{2}}}
\end{array}\right]\left[\begin{array}{l}
x \\
\alpha \\
\beta \\
y
\end{array}\right],
$$

where

$$
\xi=\sqrt{c^{2} \tau^{2}+\left(c^{2}-2 a^{2}\right) \kappa^{2}-2 a b \tau} ; w=\frac{\xi}{\sqrt{a^{2}+b^{2}}}=\frac{d s^{*}}{d s}
$$


F. Almaz, M.A. Külahci - A Note on Special Smarandache Curves ...

$$
\begin{aligned}
A_{1} & =\frac{a \kappa+b \tau}{\xi}, A_{2}=\frac{-c \kappa}{\xi}, A_{3}=\frac{-c \tau}{\xi}, A_{4}=\frac{-a}{\xi} \\
C_{1} & =\frac{\xi^{\prime}(a \kappa+b \tau)+\xi\left(a \kappa^{\prime}+b \tau^{\prime}\right)-c\left(\kappa^{2}+\tau^{2}\right)}{\xi^{3}} \sqrt{a^{2}+b^{2}} \\
C_{2} & =\frac{\xi(2 a \kappa+b \tau)-c \kappa \xi^{\prime}}{\xi^{3}} \sqrt{a^{2}+b^{2}} \\
C_{3} & =\frac{\xi c\left(a \tau-\tau^{\prime}\right)-c \tau \xi^{\prime}}{\xi^{3}} \sqrt{a^{2}+b^{2}} \\
C_{4} & =\frac{c \kappa \xi+a}{\xi^{3}} \sqrt{a^{2}+b^{2}}, \\
M= & \frac{-2 a(a \kappa+b \tau)+c\left(\kappa^{2}+\tau^{2}\right)}{\xi^{2}}
\end{aligned}
$$

ii) The cone curvatures $\kappa_{\gamma_{\alpha \beta y}}\left(s^{*}\right)$ and $\tau_{\gamma_{\alpha \beta y}}\left(s^{*}\right)$ of the curve $\gamma_{\alpha \beta y}$ is given by

$$
\begin{gathered}
\kappa_{\gamma_{\alpha \beta y}}\left(s^{*}\right)=\frac{2 a(a \kappa+b \tau)-c\left(\kappa^{2}+\tau^{2}\right)}{2 \xi^{2}} \\
\tau_{\gamma_{\alpha \beta y}}^{2}\left(s^{*}\right)=2\left(C_{1}+\kappa^{\prime}\right)\left(C_{4}+\frac{c M}{2 \sqrt{a^{2}+b^{2}}}\right)+\left(C_{2}+\frac{a M}{2 \sqrt{a^{2}+b^{2}}}+\kappa\right)^{2} \\
+\left(C_{3}-\frac{b M}{2 \sqrt{a^{2}+b^{2}}}\right)^{2}-\kappa^{2} .
\end{gathered}
$$

where

$$
s^{*}=\frac{1}{\sqrt{a^{2}+b^{2}}} \int \sqrt{c^{2} \tau^{2}+\left(c^{2}-2 a^{2}\right) \kappa^{2}-2 a b \tau} . d s ; a, b, c \in \mathbb{R}_{0}^{+} .
$$

Proof. i) Differentiating the equation (3.34) with respect to $s$ and considering (2.1), we find

$$
\gamma_{\alpha \beta y}^{\prime}\left(s^{*}\right) \frac{d s^{*}}{d s}=\frac{1}{\sqrt{a^{2}+b^{2}}}((a \kappa+b \tau) x(s)+(-c \kappa) \alpha(s)+(-c \tau) \beta(s)-a y(s)) .
$$

This can be written as follows

$$
\alpha_{\alpha \beta y}\left(s^{*}\right)=\frac{a \kappa+b \tau}{\xi} x(s)+\frac{-c \kappa}{\xi} \alpha(s)+\frac{-c \tau}{\xi} \beta(s)+\frac{-a}{\xi} y(s),
$$

where

$$
\xi=\sqrt{c^{2} \tau^{2}+\left(c^{2}-2 a^{2}\right) \kappa^{2}-2 a b \tau}, \frac{d s^{*}}{d s}=\frac{Z}{\sqrt{a^{2}+b^{2}}}=W .
$$


Differentiating (3.41) and using (3.42), we get

$$
\begin{aligned}
\gamma_{\alpha \beta y}^{\prime \prime}\left(s^{*}\right)= & \frac{\sqrt{a^{2}+b^{2}}}{\xi^{3}}\left(\left(\xi^{\prime}(a \kappa+b \tau)+\xi\left(a \kappa^{\prime}+b \tau^{\prime}\right)-c\left(\kappa^{2}+\tau^{2}\right)\right) x(s)\right. \\
& +\left(\xi(2 a \kappa+b \tau)-c \kappa \xi^{\prime}\right) \alpha(s)+\left(\xi c\left(a \tau-\tau^{\prime}\right)-c \tau \xi^{\prime}\right) \beta(s) \\
& +(c \kappa \xi+a) y(s)) \\
& y_{\alpha \beta y}\left(s^{*}\right)=-\gamma_{\alpha \beta y}^{\prime \prime}-\frac{1}{2}\left\langle\gamma_{\alpha \beta y}^{\prime \prime}, \gamma_{\alpha \beta y}^{\prime \prime}\right\rangle \gamma_{\alpha \beta y} .
\end{aligned}
$$

By the help of equation (3.43), we obtain

$$
\begin{gathered}
y_{\alpha \beta y}\left(s^{*}\right)=\left(-C_{1}\right) x(s)-\left(C_{2}+\frac{a M}{2 \sqrt{a^{2}+b^{2}}}\right) \alpha(s) \\
-\left(C_{3}+\frac{b M}{2 \sqrt{a^{2}+b^{2}}}\right) \beta(s)-\left(C_{4}+\frac{c M}{2 \sqrt{a^{2}+b^{2}}}\right) y(s),
\end{gathered}
$$

where $M=\frac{-2 a(a \kappa+b \tau)+c\left(\kappa^{2}+\tau^{2}\right)}{\xi^{2}}$.

ii) Using (3.22), we have (3.36) and (3.37). where

Example 1. The curve

$$
x(s)=\frac{1}{\sqrt{2}}(\sin 2 s, \cos 2 s, 0,1)
$$

is spacelike in $\mathbf{Q}^{3}$ with arc length parameter s. Then we can write the Smarandache curves of the $x$-curve as follows:

1) $x \alpha \beta-$ Smarandache curve $\gamma_{x \alpha \beta}$ is given by

$$
\gamma_{x \alpha}(s)=\frac{1}{\sqrt{2\left(c^{2}+b^{2}\right)}}((a-4 c) \sin 2 s+2 b \cos 2 s,(a-4 c) \cos 2 s-2 b \sin 2 s, 0, a)
$$

2) $x \beta y-$ Smarandache curve $\gamma_{x \beta y}$ is given by

$$
\gamma_{x \beta y}(s)=\frac{1}{\sqrt{2\left(2 a c+b^{2}\right)}}((a-4 c) \sin 2 s-8 c \cos 2 s,(a-4 c) \cos 2 s+8 c \sin 2 s, 0, a)
$$

3) $x \alpha y-$ Smarandache curve $\gamma_{x \alpha y}$ is given by

$\gamma_{x \alpha y}(s)=\frac{1}{\sqrt{2\left(2 a c+b^{2}\right)}}(a \sin 2 s+(2 b-8 c) \cos 2 s, a \cos 2 s+(-2 b+8 c) \sin 2 s, 0, a)$

4) $\alpha \beta y-$ Smarandache curve $\gamma_{\alpha \beta y}$ is given by

$$
\gamma_{\alpha \beta y}(s)=\frac{1}{\sqrt{2\left(a^{2}+b^{2}\right)}}((a-4 b) \sin 2 s-8 c \cos 2 s,(a-4 b) \cos 2 s+8 c \sin 2 s, 0, a)
$$

where $a, b, c \in \mathbb{R}_{0}^{+}$. 
F. Almaz, M.A. Külahci - A Note on Special Smarandache Curves ...

\section{REFERENCES}

[1] H.S. Abdel-Aziz, M.K. Saad, Smarandache Curves of Some Special Curves in the Galilean 3-Space, Honan Mathematical J. 37, 2 (2015), 253-264 .

[2] A.T. Ali, Special Smarandache Curves in the Euclidean Space, International J. Mathematical Combinatorics 2 (2010), 30-36.

[3] A.T. Ali, Time-like Smarandache Curves Derived from a Space-like Helix, Journal of Dynamical Systems and Geometric Theories 8, 1 (2010), 93-100.

[4] C. Ashbacher, Smarandache Geometries, Smarandache Notions Journal 8, (1-3) (1997), 212-215.

[5] N. Bayrak, O. Bektas, S. Yuce, Special Smarandache Curves in $\mathbb{R}_{1}^{3}$, Commun. Fac. Sci. Univ. Ank. Ser. A1 Math. Stat. 65, 2 (2016), 143-160.

[6] O. Bektas, S. Yuce, Special Smarandache Curves According to Darboux Frame in $E^{3}$, Romanian Journal of Mathematics and Computer Science 3, 1 (2013), 48-59.

[7] M. Cetin, H. Kocayigit, On the Quaternionic Smarandache Curves in Euclidean 3-Space, Int. J. Contemp. Math. Sciences 8, 3 (2013), 139-150.

[8] M. Kulahci, M. Bektas, M. Ergut, Curves of $A W(k)$-Type in 3-Dimensional Null Cone, Physics Letters A 371 (2017), 275-277.

[9] M. Kulahci, F. Almaz, Some Characterizations of Osculating in the Lightlike Cone, Bol. Soc. Paran. Math. 35, 2 (2017), 39-48.

[10] H. Liu, Curves in the Lightlike Cone, Contribbutions to Algebra and Geometry 45, 1 (2014), 291-303.

[11] H. Liu, Q. Meng, Representation Formulas of Curves in a Two- and ThreeDimensional Lightlike Cone, Results Math. 59 (2011), 437-451.

[12] B. O'Neill, Semi-Riemannian Geometry with Applications to Relativity, Academic Press, London, 1983.

[13] U. Ozturk, E.B.K. Ozturk, Smarandache Curves According to Curves on Spacelike Surface in Minkowski 3-Space $\mathbb{R}_{1}^{3}$, Journal of Discrete Mathematics, vol. 2014, article ID829581, 10 pages.

[14] U. Ozturk, E.B.K Ozturk, K. Ilarslan, E. Nesovic, On Smarandache Curves Lying in Lightcone in Minkowski 3-Space, Journal of Dynamical Systems and Geometric Theories 12, 1 (2014), 81-91.

[15] S. Senyurt, A. Caliskan, Smarandache Curves in Terms of Sabban Frame of fixed Pole Curve, Bol. Soc. Paran. Math. 34, 2 (2016), 53-62.

[16] M. Turgut, S. Yilmaz, Smarandache Curves in Minkowski Spacetime, International Journal of Mathematical Combinatorics 3 (2018), 51-55. 
F. Almaz, M.A. Külahci - A Note on Special Smarandache Curves ...

Fatma Almaz,

Department of Mathematics, Faculty of Science, Firat University,

\section{ELAZIĞ, TURKEY}

email: fb_fat_almaz@hotmail.com

Mihriban Alyamaç Külahcı

Department of Mathematics, Faculty of Science, Firat University, ELAZIĞ, TURKEY

email: mihribankulahci@gmail.com 